\title{
The gut-microbiome-brain connection
}

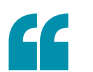

\section{alterations in}

commensal

gut bacteria

contribute

to certain

behavioural

abnormalities

in a mouse

model of ASD

Studies in mice and human patients suggest that microbial imbalances in the gut influence various social, emotional and anxiety-like behaviours. Interestingly, gastrointestinal (GI) problems are common in autism spectrum disorders (ASDs), which are associated with social interaction deficits and stereotypical behaviours. A new study in Cell provides evidence that alterations in commensal gut bacteria contribute to certain behavioural abnormalities in a mouse model of ASD, highlighting the potential benefit of probiotic therapies for some of the features of such disorders.

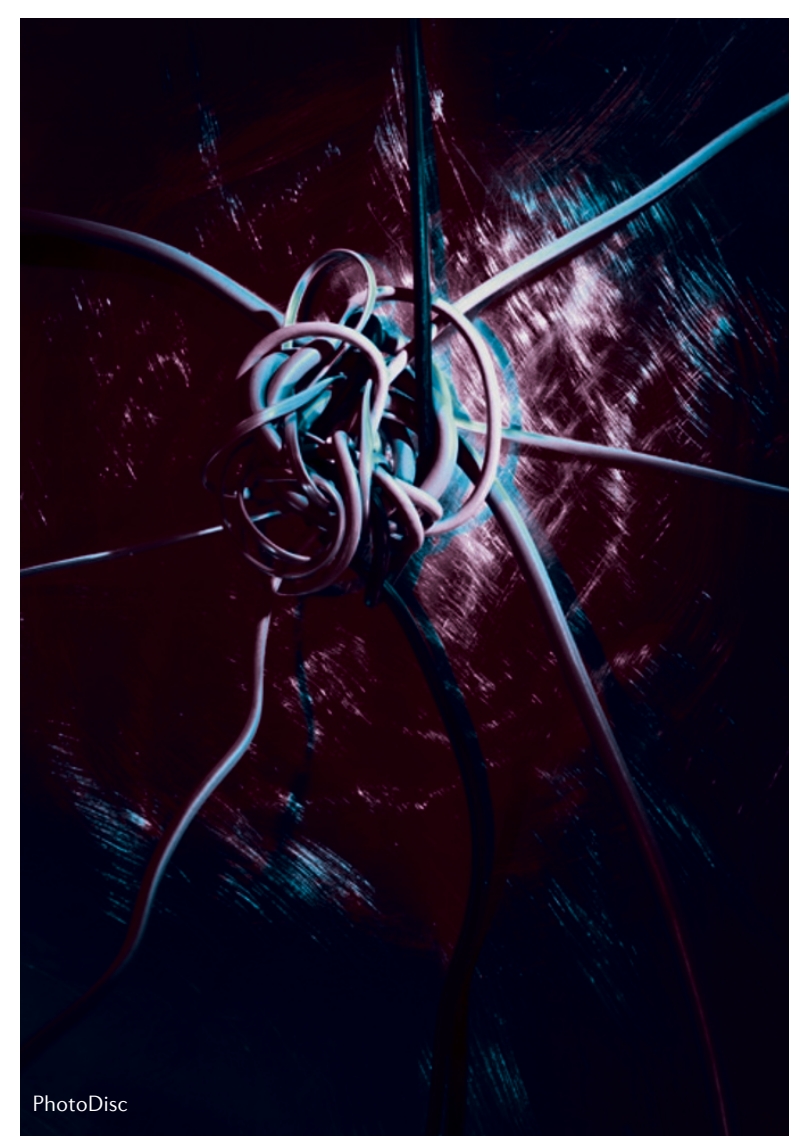

Hsiao et al. studied the offspring of the maternal immune activation (MIA) mouse model, which is used to investigate the link between maternal infection and ASDs. MIA offspring exhibited certain behavioural and neuropathological symptoms that are reminiscent of ASDs, and the authors found that, by 3 weeks of age, these mice also showed an increase in intestinal permeability and an abnormal intestinal cytokine profile that was characterized by high levels of the inflammatory marker interleukin-6.

Further experiments showed that the guts of MIA offspring harbour different strains of Clostridia and Bacteroidia, the two major classes of commensal gut bacteria, compared with wild-type mice, which is consistent with studies in individuals with ASD. Treatment with Bacteroides fragilis has been shown to restore intestinal permeability in an experimental colitis model, so the authors fed the MIA offspring such bacteria every other day, for 6 days at weaning and examined the GI tract 8 weeks later. B. fragilis treatment not only corrected the defects in GI barrier integrity but also restored the altered cytokine profile and microbiome composition.

Moreover, the treatment ameliorated some of the ASD-related behavioural abnormalities that the MIA offspring mice exhibit. For example, treatment led to decreases in anxietylike and stereotyped behaviours during open-field exploration and marble-burying assays, respectively, and to an increase in the number of ultrasonic vocalizations produced in response to social encounters. However, despite treatment with
B. fragilis, MIA offspring retained defects in sociability and social preference, suggesting that the treatment modulates only some of the circuits underlying the behavioural impairments that characterize ASDs.

Finally, in an attempt to uncover the mechanism through which $B$. fragilis exerts its effects on the gut and brain, the authors examined the levels of serum metabolites in MIA offspring. Several metabolites were markedly increased in these mice and were restored to normal levels by $B$. fragilis. One of them, 4-ethylphenylsulphate (4EPS), which is produced by commensal bacteria, was increased 46-fold in the untreated mice. Systemic administration of 4EPS induced anxiety-like behaviour in naïve mice, which is similar to that observed in MIA offspring. Other ASD-related behavioural abnormalities were not observed, suggesting that further studies on these metabolites could uncover other molecules that may mediate distinct effects of GI abnormalities on behaviour.

Although most of the mechanistic details by which the gut microbiome can affect behaviour remain to be established, the possibility of treating behavioural abnormalities related to ASDs (and perhaps other disorders that are characterized by behavioural abnormalities, such as schizophrenia, obsessive compulsive disorder or Angelman syndrome) with microbebased therapies is likely to attract a lot of attention.

Monica Hoyos Flight

ORIGINAL RESEARCH PAPER Hsiao, E. Y. et al.

Microbiota modulate behavioral and physiological abnormalities associated with neurodevelopmental disorders. Cell http://dx.doi. org/10.1016/j.cell.2013.11.024 (2013) 